\title{
Particle motion in a deformed potential using a transformed oscillator basis
}

\author{
J. A. Lay, ${ }^{*}$ A. M. Moro, ${ }^{\dagger}$ and J. M. Arias ${ }^{\ddagger}$ \\ Departamento de FAMN, Facultad de Física, Universidad de Sevilla, Apartado 1065, E-41080 Sevilla, Spain \\ J. Gómez-Camacho ${ }^{\S}$ \\ Departamento de FAMN, Facultad de Física, Universidad de Sevilla, Apartado 1065, E-41080 Sevilla, Spain and \\ Centro Nacional de Aceleradores, Avenida Thomas A. Edison, E-41092 Sevilla, Spain \\ (Received 23 February 2012; revised manuscript received 17 April 2012; published 22 May 2012)
}

\begin{abstract}
The quantum description of a particle moving in a deformed potential is investigated. A pseudostate (PS) basis is used to represent the states of the composite system. This PS basis is obtained by diagonalizing the system Hamiltonian in a family of square integrable functions. In this work the transformed harmonic oscillator (THO) functions, obtained from the solutions of the harmonic oscillator using a local scale transformation (LST), are used. The proposed method is applied to the ${ }^{11} \mathrm{Be}$ nucleus, treated in a two-body model $\left({ }^{10} \mathrm{Be}+n\right)$. Structure observables have been studied. Wave functions and energies obtained for the bound states and some low-lying resonances are compared with those obtained by direct integration of the Schrödinger equation. The dipole and quadrupole electric transition probabilities for the low-energy continuum have been calculated in the THO basis, and compared with the exact distributions obtained with the scattering states.
\end{abstract}

DOI: 10.1103/PhysRevC.85.054618

PACS number(s): 24.10.Eq, 25.10.+s, 25.45.De

\section{INTRODUCTION}

Nuclei in the proximity of the proton and neutron drip lines are often weakly bound, or even unbound, and hence their properties are influenced by positive-energy states. Collisions of these systems by stable nuclei will be also influenced by the coupling to the unbound states. This effect was first noticed in deuteron-induced reactions, and later observed in the scattering of other loosely bound nuclei, such as halo nuclei. For weakly-bound nuclei with a two-body or three-body structure, the effect of the coupling to the unbound states has been successfully described within the continuum-discretized coupled-channels (CDCC) method [1,2].

The description of the positive-energy states can be done in terms of scattering states, obtained by direct integration of the Schrödinger equation with the appropriate boundary conditions, and this was indeed the original choice of the CDCC method. Alternatively, the bound and unbound states of the system can be obtained by diagonalizing the Hamiltonian in a suitable basis of square-integrable functions. The eigenfunctions of the system are expressed as an expansion in the basis functions. Due to the truncation of the basis required in any practical calculation, these eigenstates and their corresponding eigenvalues can be regarded as a finite approximation to the exact states of the system and are referred hereafter as pseudostates (PS). This procedure has been applied, for example, to describe the scattering of a two-body nucleus [3-5] and, more recently, also to the scattering of three-body nuclei [6-9]. A variety of bases have been used in these applications, such as harmonic oscillator (HO), Gaussian, Laguerre functions, etc.

\footnotetext{
*lay@us.es

†'moro@us.es

†ariasc@us.es

§gomez@us.es
}

The aforementioned applications ignore possible excitations of the cluster constituents. However, recent experimental and theoretical developments indicate that deformation can play a significant role in the structure and dynamics of these proton or neutron rich nuclei, such as halo nuclei containing a deformed core. The description of the continuum states of these systems can still be done using scattering states [10]. For example, the effect of core excitation in the scattering of a two-body halo nucleus has been recently studied in a extension of the CDCC method [11] and, more recently, in the distorted-wave Born approximation (DWBA) model proposed in Refs. [12,13].

As in the spherical case, continuum states of a deformed system can be also described using a PS basis. A natural choice for the PS would be the deformed HO potential $[14,15]$. However, this basis is not suitable to describe the bound states of weakly bound nuclei due to its Gaussian asymptotic behavior. Several alternatives have been proposed in the literature, for example, the eigenstates of a truncated Woods-Saxon potential [16] or the Sturmian basis $[17,18]$.

In this work, we propose the use of a transformed harmonic oscillator (THO) basis to describe the states of a two-body system mutually interacting with a deformed potential. This basis has been previously applied to the case of spherical systems [5], so we present here its extension to deformed systems. The THO basis is obtained by applying a local scale transformation (LST) to the harmonic oscillator (HO) basis. The LST, adopted from a previous work of Karataglidis et al. [19], is such that it transforms the Gaussian asymptotic behavior into an exponential form, thus ensuring the correct asymptotic behavior for the bound wave functions. The accuracy of this THO basis was tested for several reactions induced by deuteron and halo nuclei, showing an excellent agreement with the standard binning method, and an improved convergence rate in the case of narrow resonances $[5,20]$. 
For a deformed potential, the calculation of bound and unbound states becomes a multichannel problem, since, in general, for each physical state there will be contributions from several orbital angular momenta and core states. For bound states, the calculation of the energies and eigenfunctions is analogous to the single-channel case, because these quantities are directly obtained from the diagonalization in the chosen PS basis. For unbound states, the eigenfunctions (and their corresponding eigenvalues) obtained from the Hamiltonian diagonalization can be regarded as a finite and discrete representation of the exact states. In general, resonances (quasistationary states) correspond to combinations of these positive-energy eigenstates, and hence their identification is not straightforward.

For a particle moving in a central potential (with possibly a spin-orbit component) this is a relatively straightforward problem, and indeed a variety of methods have been proposed to compute resonance energies and widths. For example, they can be obtained from the poles of the $S$ matrix in the complex energy plane. A simpler method is to define the resonance as the energy at which the phase shift crosses $\pi / 2$. The width is then obtained from the inverse of the derivative of the phase shift, evaluated at the energy of the resonance. These methods rely on the knowledge of the scattering states at large distances (from which the $S$ matrix and hence the phase shifts can be extracted) and then they cannot be directly applied to PS methods, given the wrong asymptotic behaviour of the PS functions. In this case, the identification of resonances can be done using the so-called stabilization method [21,22]. This is a procedure devised to identify and construct the most localized continuum wave functions when the positive energy states are expanded in a discrete basis, depending on one or more parameters. In practice, this can be achieved by diagonalizing the Hamiltonian as a function of these parameters (for example, the basis size) and then scanning the resultant eigenvalues for the continual appearance of a stabilized value which, unlike the others, is insensitive to the size of the basis. In some previous works, we have successfully applied this technique to obtain the resonances of two-body systems with central potentials using the THO basis $[5,20]$. In this work, we explore the validity of this method for the multichannel situation that arises in the deformed case. Our aim with this work is to assess the capability of the THO basis for calculations including core deformation in the simpler two-body systems, such as ${ }^{11} \mathrm{Be}$. This step is necessary and unavoidable for providing a solid foundation to proceed with the generalization of the formalism to more challenging situations, such as the case of three-body composite systems including core deformation, or to the scattering of a two-body system by a third body, including core deformation in one of the clusters of the composite system.

The work is structured as follows. In Sec. II the THO method based on the parametric LST is reviewed and the structure model used in subsequent calculations is discussed. In Sec. III, general expressions for the electric transition operators for the particular case of a two-body system with a deformed core are provided. In Sec. IV the model is applied to describe the structure of the ${ }^{11} \mathrm{Be}$ nucleus. Finally, in Sec. V the main results of this work are summarized.

\section{EIGENSTATES OF A DEFORMED POTENTIAL IN A PS BASIS: THE THO BASIS}

In this section, we briefly review the features of the PS basis used in this work. This basis is an extension of the THO basis used in our previous works to describe the states of a composite system consisting of two interacting inert fragments, such as a valence particle (proton/neutron) and a spherical and stable core. The goal of this extension is to allow core-excited admixtures in the description of the states of the composite system and hence the possibility of dynamic core excitation mechanisms in reactions involving these nuclei. For completeness, we review first the situation in which the core degrees of freedom are neglected. In this case, the core + valence Hamiltonian is simply given by

$$
H=T_{r}+V_{v c}(\vec{r}),
$$

where $\vec{r}$ is the relative coordinate between the valence and the core, $T_{r}$ is the core-valence kinetic energy operator, and $V_{v c}(\vec{r})$ is the interaction between the valence particle and the core. The eigenstates of this Hamiltonian can be characterized by the energy eigenvalues $(\varepsilon)$ and the set of quantum numbers $\{\ell, s, j\}$, which correspond to the orbital angular momentum $(\ell)$, the valence spin $(s)$ and their sum $(\vec{j}=\vec{\ell}+\vec{s})$. For a central potential with, possibly, a spin-orbit term, these states can be written as

$$
\phi_{\varepsilon, \ell, j}(\vec{r})=R_{\varepsilon, \ell, j}(r) \mathcal{Y}_{\ell s j m}(\hat{r}),
$$

where $\mathcal{Y}_{\ell s j m}(\hat{r})=\left[Y_{\ell}(\hat{r}) \otimes \chi_{s}\right]_{j m}$, with $\chi_{s}$ being a spin function. The radial functions $R_{\varepsilon, \ell, j}(r)$ can be obtained by solving the Schrödinger equation subject to the appropriate boundary condition for bound $(\varepsilon<0)$ or unbound $(\varepsilon>0)$ states. Alternatively, these functions can be obtained by diagonalizing the Hamiltonian (1) in a discrete basis. Since any complete basis will be infinite, this procedure is not feasible in practice unless the basis is truncated. By doing so, one obtains a finite (and approximated) expansion of the functions $R(r)$ in the selected basis. If the basis functions are denoted by $\varphi_{n, \ell, j}(\vec{r})=\chi_{n, \ell}(r) \mathcal{Y}_{\ell s j m}(\hat{r})$, we will have

$$
R_{\beta}(r)=\sum_{n=1}^{N} c_{\beta, n} \chi_{n, \ell}(r),
$$

where $\beta \equiv\{\varepsilon, \ell, s, j\}$ and $N$ is the number of states retained in the basis.

As already mentioned, there are many possible choices for the basis functions $\left\{\varphi_{n}\right\}$ (Gaussians, harmonic oscillator, Laguerre, etc). In this work we use the transformed harmonic oscillator (THO) basis, obtained from the harmonic oscillator basis with an appropriate LST $[23,24]$. If the LST function is denoted by $s(r)$, the THO states are obtained as

$$
R_{n, \ell}^{\mathrm{THO}}(r)=\sqrt{\frac{d s}{d r}} R_{n, \ell}^{\mathrm{HO}}[s(r)],
$$

where $R_{n, \ell}^{\mathrm{HO}}(s)$ is the radial part of the $\mathrm{HO}$ functions. With the criterion given above, the LST is indeed not unique. In Ref. [25] the LST was defined in such a way that the first HO state is exactly transformed into the exact ground-state wave function, assuming that this is known. Therefore, by 
construction, this wave function is exactly recovered for any arbitrary size of the basis. In a more recent work [5] we adopted the parametric form of Karataglidis et al. [19],

$$
s(r)=\frac{1}{\sqrt{2} b}\left[\frac{1}{\left(\frac{1}{r}\right)^{m}+\left(\frac{1}{\gamma \sqrt{r}}\right)^{m}}\right]^{\frac{1}{m}}
$$

that depends on the parameters $m, \gamma$, and the oscillator length $b$. Note that, asymptotically, the function $s(r)$ behaves as $s(r) \sim \frac{\gamma}{b} \sqrt{\frac{r}{2}}$ and hence the functions obtained by applying this LST to the HO basis behave at large distances as $\exp \left(-\gamma^{2} r / 2 b^{2}\right)$. Therefore, the ratio $\gamma / b$ can be related to an effective linear momentum, $k_{\text {eff }}=\gamma^{2} / 2 b^{2}$, which governs the asymptotic behavior of the THO functions. As the ratio $\gamma / b$ increases, the radial extension of the basis decreases and, consequently, the eigenvalues obtained upon diagonalization of the Hamiltonian in the THO basis tend to concentrate at higher excitation energies. Therefore, $\gamma / b$ determines the density of eigenstates as a function of the excitation energy. In all the calculations presented in this work, the power $m$ has been taken as $m=4$. This choice is discussed in Ref. [19] where the authors found that the results are weakly dependent on $m$.

Note that, by construction, the family of functions $R_{n, \ell}^{\mathrm{THO}}(r)$ are orthogonal and constitute a complete set with the following normalization:

$$
\int_{0}^{\infty} r^{2}\left|R_{n, \ell}^{\mathrm{THO}}(r)\right|^{2} d r=1 .
$$

Moreover, they decay exponentially at large distances, thus ensuring the correct asymptotic behavior for the bound wave functions. In practical calculations a finite set of functions (4) is retained, and the internal Hamiltonian of the composite system is diagonalized in this truncated basis with $N$ states, giving rise to a set of eigenvalues and their associated eigenfunctions, denoted respectively by $\left\{\varepsilon_{n}\right\}$ and $\left\{\varphi_{n, \ell}^{(N)}(r)\right\}(n=1, \ldots, N)$. As the basis size is increased, the eigenstates with negative energy will tend toward the exact bound states of the system, while those with positive eigenvalues can be regarded as a finite representation of the unbound states.

The formalism can be extended to the situation in which the core degrees of freedom are taken into account explicitly. In this case, the Hamiltonian (1) is generalized to

$$
H=T_{r}+V_{v c}(\vec{r}, \vec{\xi})+h_{\text {core }}(\vec{\xi}),
$$

where $h_{\text {core }}(\vec{\xi})$ is the intrinsic Hamiltonian of the core, whose eigenstates will be denoted by $\left\{\phi_{I M_{I}}\right\}$. Additional quantum numbers, required to fully specify the core states, are not included for notation simplicity. Note that the valence-core interaction, $V_{v c}(\vec{r}, \vec{\xi})$, contains now a dependence on the core degrees of freedom (denoted generically by $\vec{\xi}$ ).

The eigenstates of the Hamiltonian can no longer be written in the form of Eq. (2). Instead, these states will be a superposition of several valence configurations and core states, i.e.,

$$
\Psi_{\varepsilon ; J M}(\vec{r}, \vec{\xi})=\sum_{\alpha} R_{\varepsilon, \alpha}(r)\left[\mathcal{Y}_{\ell s j}(\hat{r}) \otimes \phi_{I}(\vec{\xi})\right]_{J M}
$$

Upon replacement of the expansion (8) into the Schrödinger equation, one gets a coupled set of differential equations for the radial functions $R_{\varepsilon, \alpha}(r)$. For bound states, these radial functions decay exponentially for $r \rightarrow \infty$ giving rise to square-integrable functions. For continuum states, the functions $R_{\varepsilon, \alpha}(r)$ are also obtained by solving a set of coupled radial equations, but subject to the boundary condition that incident waves occur only in the entrance channel characterized by a given set of quantum numbers $\alpha=\{\ell, s, j, I\}$. Therefore, for each continuum energy, there are as many scattering solutions as possible values of $\alpha$, compatible with the total angular momentum $J$.

Alternatively, the functions $R_{\varepsilon, \alpha}(r)$ can be obtained using an expansion in a PS basis, such as the THO basis described above. In this case, the basis must include also the new core degree of freedom,

$$
\Phi_{n, J M}^{\alpha}(\vec{r}, \vec{\xi})=R_{n, \alpha}^{\mathrm{THO}}(r)\left[\mathcal{Y}_{\ell s j}(\hat{r}) \otimes \phi_{I}(\vec{\xi})\right]_{J M}
$$

In this basis, the states of the system will be expressed as

$$
\Psi_{i, J M}^{(N)}(\vec{r}, \vec{\xi})=\sum_{n=1}^{N} \sum_{\alpha} c_{n, \alpha, J}^{i} \Phi_{n, J M}^{\alpha}(\vec{r}, \vec{\xi}),
$$

where $i$ is an index that labels the order of the eigenstate.

These eigenstates are spread in the energy spectrum with a density of states which relies on the basis parameters, mainly $N$ and $\gamma / b$, and on the continuum structure for the selected Hamiltonian, i.e., the presence of resonances or different breakup thresholds. Moreover, this density reflects the momentum distribution of the eigenstates which becomes important in order to obtain continuous energy or momentum distributions of different observables from their discrete representation in the PS basis [3,5,20,26]. Generalizing the expression in Ref. [20], the density of states is here defined as

$$
\rho(k)=\sum_{i=1}^{N} \sum_{\alpha}^{n_{\alpha}}\left\langle k_{\alpha} J_{f} \mid \Psi_{i, J M}^{(N)}\right\rangle,
$$

where $\left|k_{\alpha} J_{f}\right\rangle$ denotes the exact scattering wave function for an incoming wave in the $\alpha$ channel. Note that the difference between $k$ and $k_{\alpha}$ relies on the threshold energy for each channel.

With this definition the integral of the density with respect to the momentum is the number of THO functions selected $(N)$ times the number of channels $\left(n_{\alpha}\right)$ :

$$
\int_{0}^{\infty} \rho(k) d k=N n_{\alpha}
$$

assuming that we have included $N$ THO functions for each channel $\alpha$. Note that this integrated density is independent of the LST parameters.

The aforementioned method can be applied to any Hamiltonian of the form (7). In the calculations presented in this work, the composite system is treated within the particle-rotor model [27]. Therefore, we assume that the core nucleus has a permanent deformation which, for simplicity, is taken to be axially symmetric. Thus, we can characterize the deformation by a single parameter $\beta_{2}$. In the body-fixed frame, the surface radius is then parametrized as $R(\hat{\xi})=R_{0}\left[1+\beta_{2} Y_{20}(\hat{\xi})\right]$, 
with $R_{0}$ being an average radius. Starting from a central potential, $V_{v c}^{(0)}(r)$, the full valence-core interaction is obtained by deforming this interaction as

$$
V_{v c}(\vec{r}, \hat{\xi})=V_{v c}^{(0)}\left(r-\delta_{2} Y_{20}(\hat{\xi})\right),
$$

with $\delta_{2}=\beta_{2} R_{0}$ being the deformation length. Transforming to the space-fixed frame of reference, and expanding in spherical harmonics, this deformed potential reads

$$
V_{v c}(\vec{r}, \vec{\xi})=\sum_{\mathcal{L}, \mathcal{M}} V_{v c}^{(\mathcal{L})}(r) Y_{\mathcal{L M}}(\hat{r}) Y_{\mathcal{L} \mathcal{M}}^{*}(\hat{\xi}),
$$

where the radial form factors $V_{v c}^{(\mathcal{L})}(r)$ are obtained by projecting the deformed potential (13) onto the required multipoles.

\section{ELECTRIC TRANSITION PROBABILITIES IN THE PS BASIS}

The accuracy of the PS basis to represent the continuum can be studied by comparing the ground-state-to-continuum transition probability due to a given operator. Here we consider the important case of the electric dissociation of the initial nucleus into the fragments $c+v$. This involves a matrix element between a bound state (typically the ground state) and the continuum states.

The electric transition probability between two bound states $\left|J_{i}\right\rangle$ and $\left|J_{f}\right\rangle$ (assumed here to be unit normalized) is given by the reduced matrix element (according to Brink and Satchler convention [28])

$$
\mathcal{B}(E \lambda ; i \rightarrow f)=\frac{2 J_{f}+1}{2 J_{i}+1}\left|\left\langle J_{f} \| \mathcal{M}(E \lambda)|| J_{i}\right\rangle\right|^{2},
$$

where $\mathcal{M}$ is the multipole operator. In a core + valence model, the electric transition operator can be written as a sum of three terms [29]: one for the excitation of the valence particle outside the core, one for the excitation of the core as a whole, and one for mixed excitations involving simultaneous excitations of core and valence particle,

$$
\begin{aligned}
\mathcal{M}(E \lambda \mu)= & \sum_{k=1}^{\lambda-1} \sum_{m=-k}^{k} f_{\lambda}(k, m, \mu) \\
& \times \mathcal{M}_{s p}(E k m) \mathcal{M}_{\text {core }}(E(\lambda-k)(\mu-m)) \\
& +\mathcal{M}_{s p}(E \lambda \mu)+\mathcal{M}_{\text {core }}(E \lambda \mu),
\end{aligned}
$$

where $f_{\lambda}(k, m, \mu)$ is a well defined function of its indices and the single-particle contribution has the usual form,

$$
\mathcal{M}_{s p}(E \lambda \mu)=Z_{\mathrm{eff}}^{(\lambda)} e r^{\lambda} Y_{\lambda \mu}(\hat{r}),
$$

with the effective charge

$$
Z_{\mathrm{eff}}^{(\lambda)}=Z_{v}\left(\frac{m_{c}}{m_{v}+m_{c}}\right)^{\lambda}+Z_{c}\left(-\frac{m_{v}}{m_{v}+m_{c}}\right)^{\lambda} .
$$

In the case of a transition to a continuum of states, $\left|k J_{f}\right\rangle$, the definition (15) is replaced by (see, for example, Ref. [30])

$$
\frac{d \mathcal{B}(E \lambda)}{d \varepsilon}=\frac{2 J_{f}+1}{2 J_{i}+1} \frac{\mu_{v c} k}{(2 \pi)^{3} \hbar^{2}}\left|\left\langle k J_{f}\|\mathcal{M}(E \lambda)\| J_{i}\right\rangle\right|^{2},
$$

with $k=\sqrt{2 \mu_{v c} \varepsilon} / \hbar$. Note that the extra factor appearing in Eq. (19) with respect to Eq. (15) is consistent with the convention $\left\langle k J \mid k^{\prime} J\right\rangle=\delta\left(k-k^{\prime}\right)$ and the asymptotic behavior,

$$
\begin{aligned}
u_{\alpha^{\prime}}\left(k_{\alpha^{\prime}}, r\right) \stackrel{r \rightarrow \infty}{\longrightarrow} & \frac{1}{2} i e^{2 i \sigma_{\ell^{\prime}}}\left[\delta_{\alpha^{\prime} \alpha} H_{\ell}^{*}\left(k_{\alpha} r\right)\right. \\
& \left.-\left(\frac{v_{\alpha}}{v_{\alpha^{\prime}}}\right)^{\frac{1}{2}} S_{\alpha^{\prime}, \alpha}^{(J)} H_{\ell^{\prime}}\left(k_{\alpha^{\prime}} r\right)\right],
\end{aligned}
$$

where $u_{\alpha}\left(k_{\alpha}, r\right)=R_{\alpha}\left(k_{\alpha}, r\right) r$ (using an obvious notation where the continuum $\varepsilon$ label has been replaced by a dependence on the corresponding momentum $k$ ).

Using a finite basis, one may calculate only discrete values for the transition probability. According to Eq. (15), the $B(E \lambda)$ between the ground state (with angular momentum $J_{i}$ ) and the $n$th PS is given by

$$
\mathcal{B}^{(N)}(E \lambda ; \text { g.s. } \rightarrow n)=\frac{2 J_{f}+1}{2 J_{i}+1}\left|\left\langle\Psi_{n, J_{f}}^{(N)}|| \mathcal{M}(E \lambda)|| \Psi_{\text {g.s. }}\right\rangle\right|^{2} .
$$

In order to relate this discrete representation to the continuous distribution (19), one may derive a continuous approximation to Eq. (19) by introducing the identity in the truncated PS basis, i.e.,

$$
I_{J M}^{(N)}=\sum_{n=1}^{N}\left|\Psi_{n, J M}^{(N)}\right\rangle\left\langle\Psi_{n, J M}^{(N)}\right| .
$$

For $N \rightarrow \infty$ this expression tends toward the exact identity operator for the Hilbert space spanned by the eigenfunctions of the considered Hamiltonian. By inserting Eq. (22) into the exact expression (19), we obtain the approximate continuous distribution

$$
\begin{aligned}
\frac{d \mathcal{B}(E \lambda)}{d \varepsilon} \simeq & \frac{2 J_{f}+1}{2 J_{i}+1} \frac{\mu_{v c} k}{(2 \pi)^{3} \hbar^{2}} \\
& \times\left|\sum_{n=1}^{N}\left\langle k J_{f} \mid \Psi_{n, J_{f}}^{(N)}\right\rangle\left\langle\Psi_{n, J_{f}}^{(N)}|| \mathcal{M}(E \lambda)|| \Psi_{\mathrm{g} . \mathrm{s} .}\right\rangle\right|^{2} .
\end{aligned}
$$

This approach provides a smoothing procedure to extract continuous distributions, as a function of the asymptotic energy $\varepsilon$ (or, equivalently, the linear momentum $k$ ), from the discrete distributions obtained with the PS basis [5,31]. This is particularly convenient in situations in which the calculation with the scattering states themselves is not possible, such as in the CDCC method.

\section{TEST EXAMPLE: APPLICATION TO ${ }^{11} \mathrm{Be}$}

\section{A. Energy spectrum and wave functions in the PS basis}

As an illustration of the formalism presented in the preceding section, we consider the ${ }^{11} \mathrm{Be}$ nucleus. This choice is motivated by the fact that this nucleus is one of the best known one-neutron halo nuclei. Many of its properties can be understood in a simple two-body model, comprising a valence neutron orbiting a ${ }^{10} \mathrm{Be}$ core. For example, the ground state $\left(1 / 2^{+}\right)$and the only bound excited state $\left(1 / 2^{-}\right)$are 
reasonably well described by $2 s_{1 / 2}$ and $1 p_{1 / 2}$ single-particle configurations, relative to the ${ }^{10} \mathrm{Be}$ (g.s.) core. Excited states in the continuum are also reasonably well described in terms of single-particle excitations of the halo neutron outside the ${ }^{10} \mathrm{Be}$ (g.s.) core. This single-particle picture has been extensively used in the literature to explain also reactions induced by this nucleus (see, for instance, Refs. [20,32,33]). However, there are also numerous experimental and theoretical findings that these low-lying states of ${ }^{11} \mathrm{Be}$ contain significant admixtures of core-excited components [12,34-36]. Consequently, an accurate description of reactions involving this nucleus requires the inclusion of its states beyond the simple single-particle picture.

In the calculations presented in this work, we use the particle-rotor model of Bohr and Mottelson with the ${ }^{11} \mathrm{Be}$ Hamiltonian of Ref. [18] (model Be12-b), which consists of a Woods-Saxon central part, with a fixed geometry $(R=$ $2.483 \mathrm{fm}, a=0.65 \mathrm{fm})$ and a parity-dependent strength $\left(V_{c}=\right.$ $-54.24 \mathrm{MeV}$ for positive parity states and $V_{c}=-49.67 \mathrm{MeV}$ for negative ones). The potential contains also a spin-orbit part, whose radial dependence is given by the derivative of the same Woods-Saxon shape, and strength $V_{\text {so }}=8.5 \mathrm{MeV}$. For the ${ }^{10} \mathrm{Be}$ core, this model assumes a permanent quadrupole deformation $\beta_{2}=0.67$. Only the ground state $\left(0^{+}\right)$and the first excited state $\left(2^{+}, E_{x}=3.368 \mathrm{MeV}\right)$ are included in the model space. For the valence-core orbital angular momentum, we consider the values $\ell \leqslant 3$.

To generate the THO basis we use the LST of Eq. (5) with $m=4, b=1.6 \mathrm{fm}$, and $\gamma=1.84 \mathrm{fm}^{1 / 2}$. The value of $b$ was determined in order to minimize the ground-state energy of ${ }^{11} \mathrm{Be}$ in a small THO basis. The factor $\gamma / b$ leads to a $k_{\text {eff }}$ compatible with a maximum excitation energy of about $10 \mathrm{MeV}$, which is enough for the calculations presented below.

Once these parameters have been fixed, the THO basis is generated for different values of $N$, the number of oscillator functions, and the convergence of different observables is studied with respect to this number. We should remark that the total number of basis functions is this number times the number of channels $n_{\alpha}$. However, the latter depends on the total angular momentum $J$ of the state under consideration, and will be the same in any method based on the angular momentum expansion of the wave functions. Therefore, we will refer to $N$ as the basis size, as we understand it is the most honest way of comparing with other methods. We find that the ground-state energy is already fully converged with a relatively small basis $(N \approx 15)$.

Within the model space used in our calculations $(I=0,2$, $\ell \leqslant 3)$, there are $n_{\alpha}=3$ channels contributing to the ground state wave function, namely $\left|{ }^{10} \mathrm{Be}\left(0^{+}\right) \otimes s_{1 / 2}\right\rangle, \mid{ }^{10} \mathrm{Be}\left(2^{+}\right) \otimes$ $\left.d_{3 / 2}\right\rangle$ and $\left|{ }^{10} \mathrm{Be}\left(2^{+}\right) \otimes d_{5 / 2}\right\rangle$. The weight of each component, which can be regarded as a spectroscopic factor, can be calculated from the coefficients in Eq. (10). In this case we find $0.86,0.02$, and 0.12 for the $s_{1 / 2}, d_{3 / 2}$, and $d_{5 / 2}$ components, respectively. In Fig. 1, we depict these radial parts of the ground-state wave function obtained from the diagonalization of the Hamiltonian in a THO basis with $N=15$ oscillator functions (dashed lines). For comparison, we include also the solutions obtained by direct integration of the Schrödinger equation (solid lines). Both calculations give

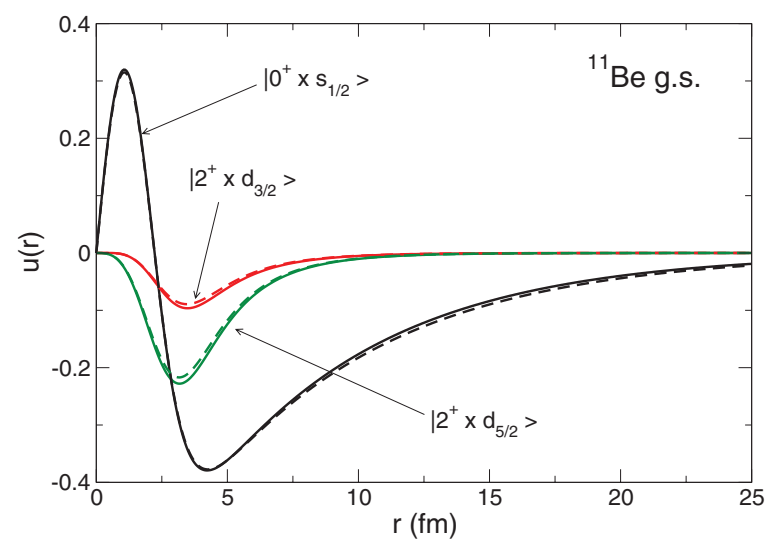

FIG. 1. (Color online) Radial parts of the ground-state wave function for the ${ }^{11} \mathrm{Be}$ nucleus obtained by direct integration of the Schrödinger equation (solid lines) and by diagonalization in a THO basis with $N=15$ states (dashed lines).

basically identical results. It can be seen, as expected, that the $\left|{ }^{10} \mathrm{Be}\left(0^{+}\right) \otimes s_{1 / 2}\right\rangle$ component is the dominant one, accounting for about $80 \%$ of the norm. This radial component exhibits a node, due to the presence of a Pauli forbidden state (arising from the $1 s_{1 / 2}$ orbital in the spherical basis).

The assumed Hamiltonian reproduces also the position of the bound excited state at $E_{x}=320 \mathrm{keV}\left(1 / 2^{-}\right)$. Indeed, this state appears also in the diagonalization of the THO basis. The separation energy is reproduced within a few percent with a basis of $N=15$ states and the radial components are also found to be in perfect agreement with those obtained by direct integration of the coupled differential equations. This is shown in Fig. 2. We proceed to discuss now the description of resonances in the PS basis. As explained in the Introduction, the identification of the resonances is done using the stabilization method of Hazi and Taylor [21,22], extended to the multichannel case. The procedure is the same as in the single-channel case, i.e., we diagonalize the Hamiltonian over either a successively larger basis set or as a function of a continuous parameter which defines the basis for a given $N$ value. Then, the evolution of the spectrum as a function of $N$

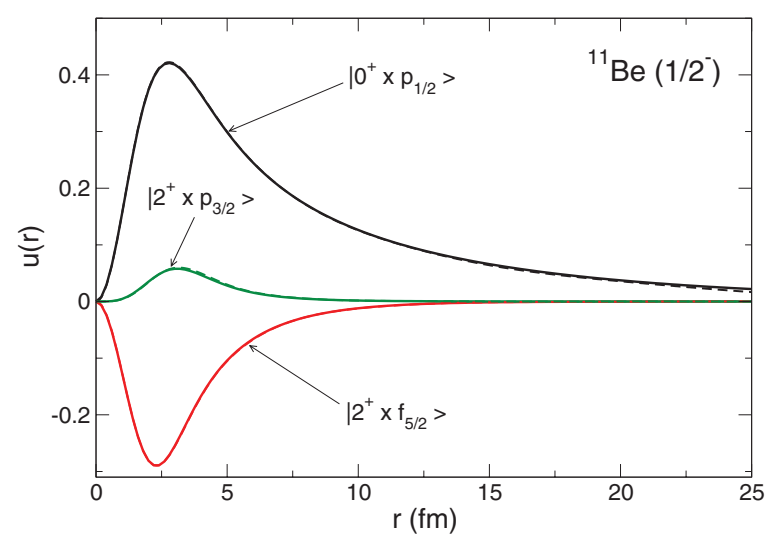

FIG. 2. (Color online) Radial parts of the $1 / 2^{-}$excited boundstate wave function for the ${ }^{11} \mathrm{Be}$ nucleus obtained by direct integration of the Schrödinger equation (solid lines) and by diagonalization in a THO basis with $N=15$ states (dashed lines). 
or the continuous parameter is studied. When a resonance is present, there are some eigenvalues whose energies are stabilized for a range of values of $N$ or the continuous parameter. This property has been employed empirically in many works, and a formal justification has also been provided by Lippmann and O'Malley [37].

The selected Hamiltonian contains low-lying resonances at $\varepsilon=1.2 \mathrm{MeV}\left(5 / 2^{+}\right), 2.7 \mathrm{MeV}\left(3 / 2^{-}\right)$, and $3.2 \mathrm{MeV}$ $\left(3 / 2^{+}\right)$[18]. These values are confirmed by applying the stabilization method with the THO basis, in the two ways described above. As an example, in Fig. 3, we show the results for $J^{\pi}=5 / 2^{+}$. In the upper panel, the sequence of
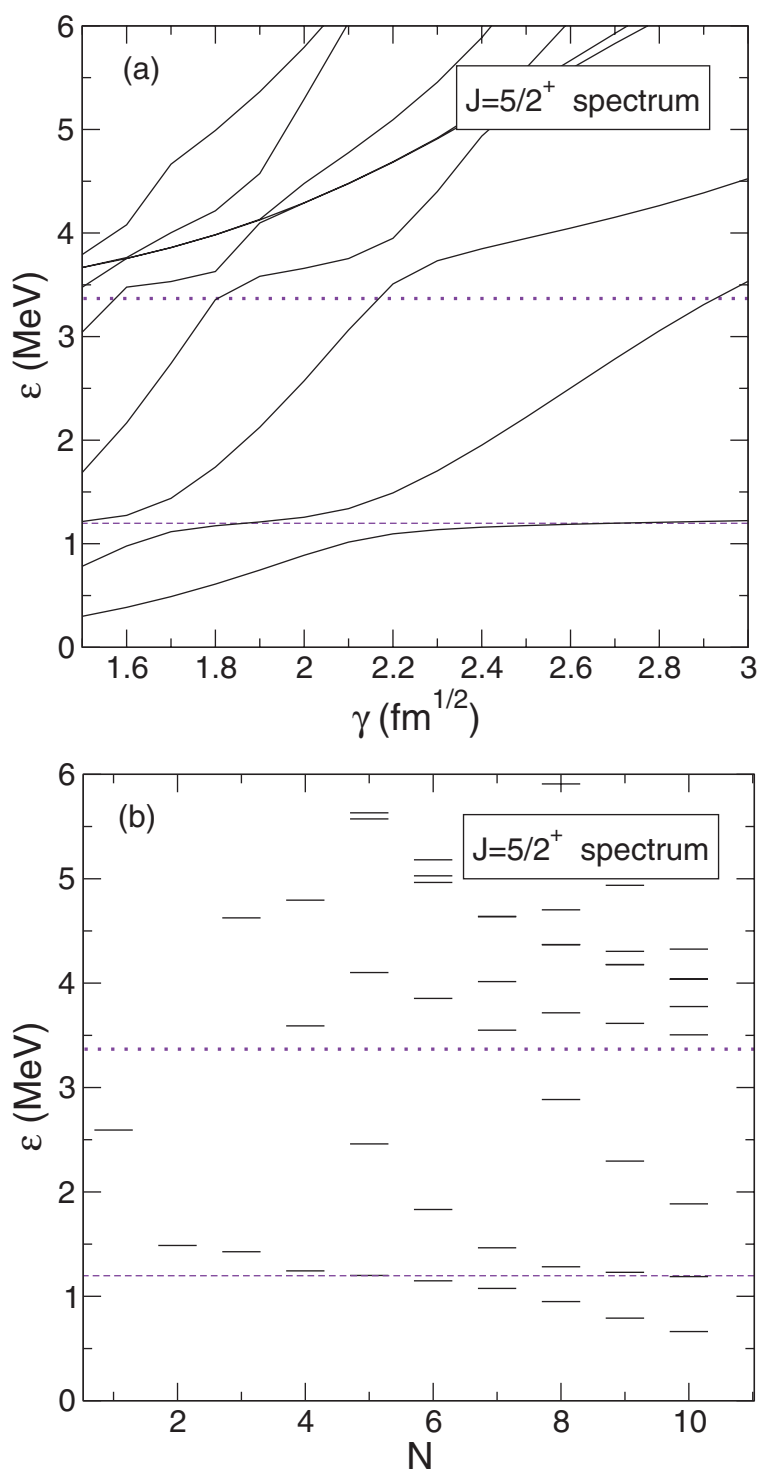

FIG. 3. (Color online) Eigenvalues obtained from the diagonalization of the ${ }^{11} \mathrm{Be}$ Hamiltonian in a THO basis, as a function of the LST continuum parameter $(\gamma)$ in the upper panel, and as a function of the number of oscillator states included in the basis in the lower panel. The dashed line indicates the energy of the $5 / 2^{+}$ resonance and the dotted line indicates the energy of the ${ }^{10} \mathrm{Be}\left(2^{+}\right)+n$ threshold. positive energy states with $J^{\pi}=5 / 2^{+}$is plotted versus the continuum parameter $\gamma$ of the LST, and for a fixed value of $N(N=10)$. In the lower panel, the $J^{\pi}=5 / 2^{+}$eigenvalues obtained from the diagonalization of the assumed Hamiltonian in the THO basis are plotted as a function of the discrete basis size parameter $(N)$, with $\gamma$ fixed to $1.84 \mathrm{fm}^{1 / 2}$. The dashed line marks the known location of the first $5 / 2^{+}$resonance deduced from the behavior of the phase shifts, and the dotted line marks the $n+{ }^{10} \mathrm{Be}\left(2^{+}\right)$threshold. In both plots, the energy stabilization precisely at the nominal energy of the resonance is apparent. Similar results are obtained for the $3 / 2^{+}$and $3 / 2^{-}$ resonances.

According to the stabilization method, the eigenfunctions corresponding to the stabilized energies should correspond to well localized states, as expected for a resonant state. From Fig. 3(b), it is apparent that for $N=5$ and $N=10$ one obtains a stabilized eigenvalue close to the known energy of the $5 / 2^{+}$resonance, i.e, $\varepsilon \approx 1.2 \mathrm{MeV}$. We have verified that the corresponding wave functions are very similar in the interior, and they reproduce very well the scattering wave function evaluated at the energy of the resonance. This is illustrated in Fig. 4(a) where we compare the radial components of the scattering wave functions, evaluated at the nominal energy of the resonance (solid lines), with the THO eigenfunction associated with the stabilized eigenvalue, for the $N=10$ basis (dashed lines). In panels (b) and (c) of the same figure, we show the equivalent calculations for the $3 / 2^{-}$and $3 / 2^{+}$resonances. In this case, the stabilized energies occur for $N=9$. Because the continuum wave functions are not square-integrable, these have been conveniently scaled for a better comparison with the PS functions.

Note that, for these three resonances, the channels corresponding to $I=2$ are effectively bound, since the energy of these resonances is below the $n+{ }^{10} \mathrm{Be}\left(2^{+}\right)$threshold. The component based on the ${ }^{10} \mathrm{Be}(\mathrm{g}$.s. $)$ is unbound but it shows the anticipated localization reminiscent of a quasistationary state. We note that, unlike the case of the bound states, we do not expect a perfect agreement between both calculations due to the exponential behavior of the PS basis at large distances. Apart from that, it is also seen that, in the interior region, the four radial components are in very good agreement with the exact solution.

The stabilization method provides also expressions for the width of the resonances in the PS basis [22]. However, these expressions were originally developed for the singlechannel case, and hence they cannot be directly applied to our case. To have an estimate of the width of the resonance we make use of the density of states, defined according to Eq. (11). This function is shown in Fig. 5 for the discussed resonances, using different values of the basis size $(N)$. It can be seen how the density increases as more channels are open above the excitation energy of the core. It can be seen also that the presence of a resonance gives rise to a peak in the density distribution. It is noticeable that the shape of the resonance is very stable with respect to the basis size. Based on these properties, we have estimated the width of the resonance from the FWHM of the corresponding peak in the density distribution. For the $5 / 2^{+}, 3 / 2^{-}$, and $3 / 2^{+}$ 

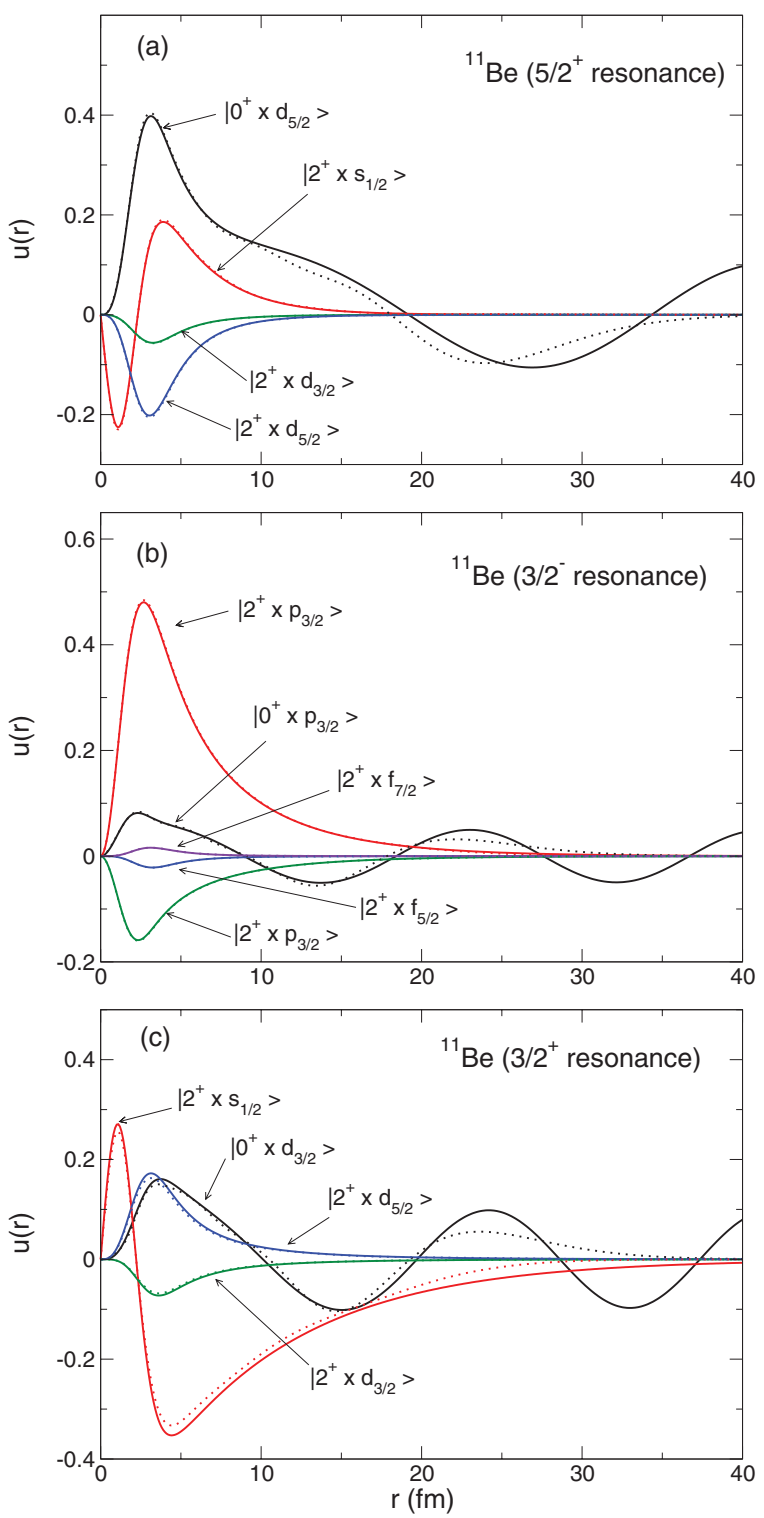

FIG. 4. (Color online) Radial parts of the continuum wave functions for the ${ }^{11} \mathrm{Be}$ resonances at $\varepsilon=1.2 \mathrm{MeV}\left(5 / 2^{+}\right), 2.7 \mathrm{MeV}$ $\left(3 / 2^{-}\right)$, and $3.2 \mathrm{MeV}\left(3 / 2^{+}\right)$. The solid lines are obtained by direct integration of the Schrödinger equation, whereas the dashed lines are the result of the diagonalization in a THO basis with $N=10\left(5 / 2^{+}\right)$ or $N=9\left(3 / 2^{-}, 3 / 2^{+}\right)$oscillator functions. For a better comparison, the normalization of the scattering state has been chosen in order to have the same magnitude as the discrete solution at the maximum.

resonances considered above, this method yields $\Gamma=125,40$, and $140 \mathrm{keV}$ respectively. These widths are to be compared with the values reported in Ref. [38], namely, $\Gamma=125,50$, and $100 \mathrm{keV}$. Except for the latter, for which our prescription gives a width $40 \%$ larger, the agreement between both methods is very good in the other two cases.

Just to complete our study, we show in Fig. 6 the comparison of the radial parts obtained by integration of the Schrödinger equation (solid lines) and by diagonalization in a THO basis with $N=15$ (dotted lines) for a nonresonant state in the

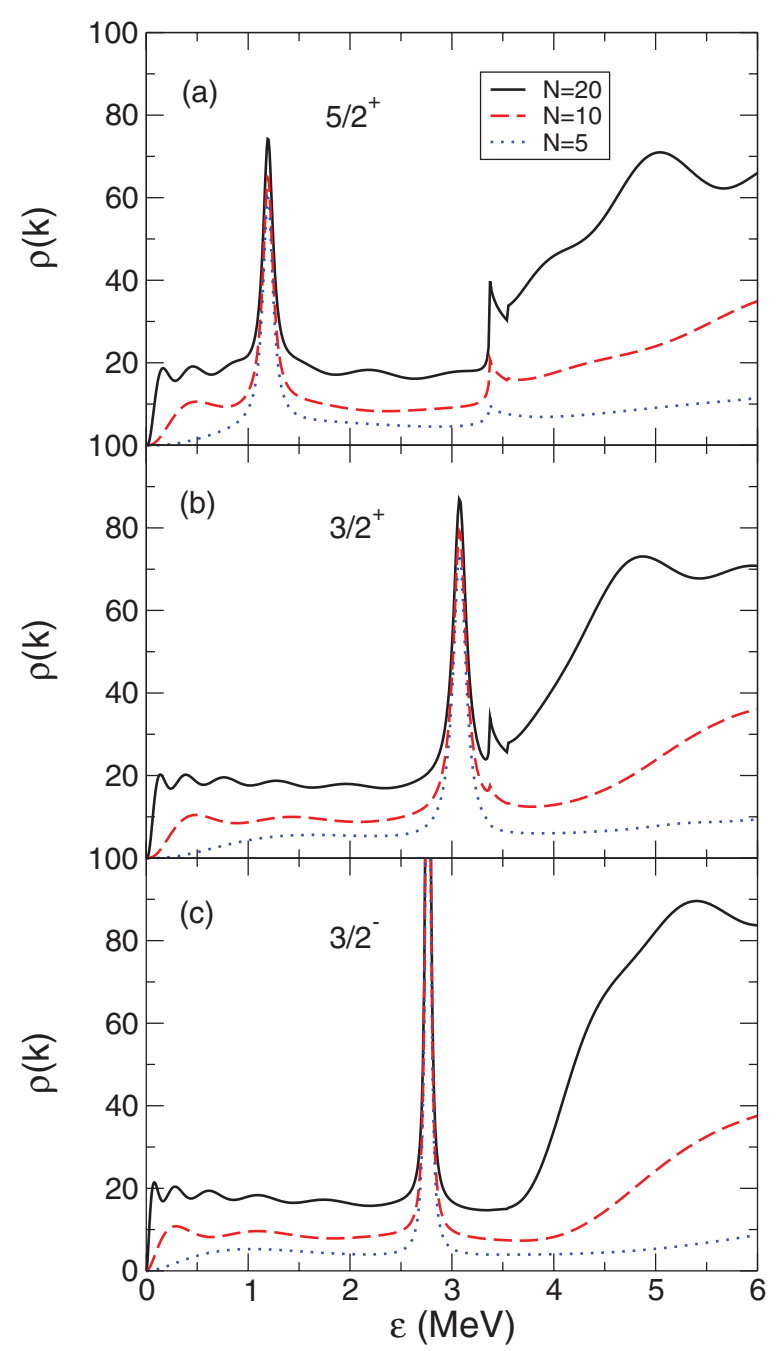

FIG. 5. (Color online) Density of states for the $5 / 2^{+}, 3 / 2^{+}$, and $3 / 2^{-}$resonances of the ${ }^{11} \mathrm{Be}$ nucleus, using different values of the basis size $N$.

continuum. It can be observed that the agreement is also good for these states.

\section{B. Electric reduced transitions probabilities}

The electric transition probabilities provide also a useful test to assess the quality of the basis to represent the continuum states. These transition probabilities can be calculated using either the exact scattering states, using Eq. (19), or the pseudostates, using Eq. (21). In the latter case, one obtains a discrete distribution, which can be converted to a continuous distribution by means of Eq. (23). In actual calculations, this equation is evaluated with a finite number of states $(N)$ and hence this formula is only approximate. The degree of agreement of this approximate formula with the exact calculation provides a measurement of the quality of the PS basis to represent the continuum for a given operator. In this section we perform this test for the $E 1$ and $E 2$ operators. 


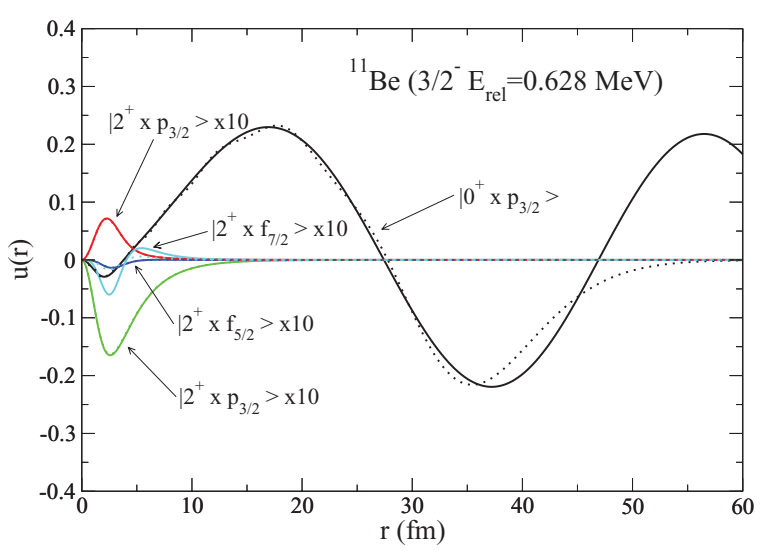

FIG. 6. (Color online) Radial parts of the $3 / 2^{-}$wave function for the ${ }^{11} \mathrm{Be}$ nucleus at $E_{\text {rel }}=0.628 \mathrm{MeV}$ obtained by direct integration of the Schrödinger equation (solid lines) and by diagonalization in a THO basis with $N=15$ states. All the components except the main one are multiplied by a factor of 10 .

According to Eq. (16), the electric operator for a valence + core system will contain in general contributions coming from the valence excitation, the core excitation, and mixed excitations. However, in our test case, ${ }^{11} \mathrm{Be}$, with core states restricted to the ground state $\left(0^{+}\right)$and the first excited state $\left(2^{+}\right)$, dipole transitions will consist of pure single-particle excitations. On the other hand, quadrupole transitions will contain both single-particle and core excitations, but not simultaneous transitions. These simultaneous transitions will only affect octupole and higher-order transitions, which will not be considered here.

In Fig. 7, the energy distribution of the $\mathcal{B}(E 1)$ obtained with a THO basis with $N=20$ functions is shown for ${ }^{11} \mathrm{Be}$. Separate contributions for $1 / 2^{-}$and $3 / 2^{-}$states are shown by dotted and dashed lines, respectively. With this basis size, the calculated THO distributions are almost indistinguishable from the exact calculation, obtained with the exact scattering

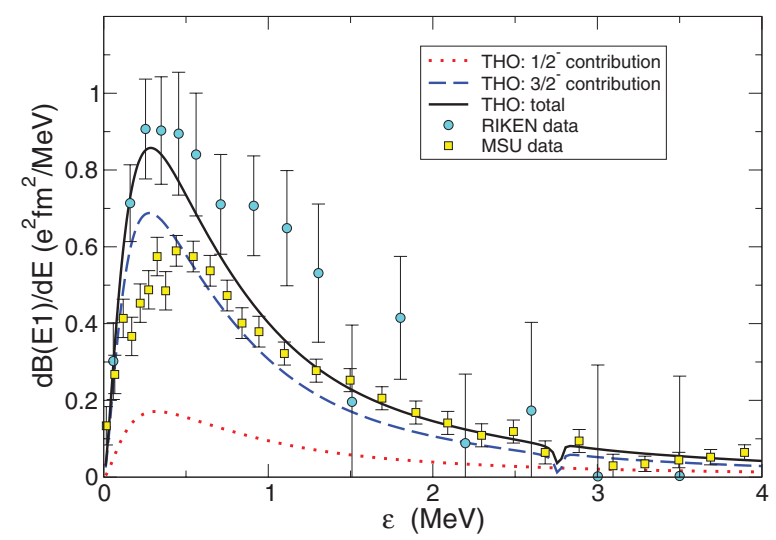

FIG. 7. (Color online) Dipole electric transition probability $\mathcal{B}(E 1)$ obtained with the THO basis and compared with experimental data from RIKEN [39] and MSU [40]. A THO basis with $N=20$ was used in the calculation, for which the calculated distribution is fully converged and is indistinguishable from the exact result using the exact scattering wave functions. states, so the latter has not been included in the figure. This agreement is not unexpected because the exponential decay of the ground-state wave function probes only the interior part of the continuum wave functions (which are well described by the PS basis) and suppresses the contribution from the asymptotic part. The available experimental distributions from two experiments performed at RIKEN [39] and MSU [40] are also shown in the plot. The theoretical distribution lies in between the two experimental sets of data. However, one has to keep in mind that the RIKEN data are inclusive with the respect to the ${ }^{10} \mathrm{Be}$ state and hence might contain contributions where the core is left in an excited state. Moreover, it is also worth noting that the calculation will be sensitive to the choice of the ${ }^{11} \mathrm{Be}$ Hamiltonian. We have not explored in this work this dependence since the purpose of this calculation is to test the quality of the basis, rather than make a detailed comparison with the data.

From Fig. 7 one sees that the calculated distribution shows a dip around $\varepsilon=2.8 \mathrm{MeV}$, which is also visible in the data from Ref. [40]. This behavior arises from the presence of the $3 / 2^{-}$ resonance at this excitation energy. This resonance is relatively narrow $(\Gamma=50 \mathrm{keV})$ but it is only weakly coupled because it is mainly built on the excited core $\left[{ }^{10} \mathrm{Be}\left(2^{+}\right)\right]$, whereas the ground state is mostly ${ }^{10} \mathrm{Be}\left(0^{+}\right)$.
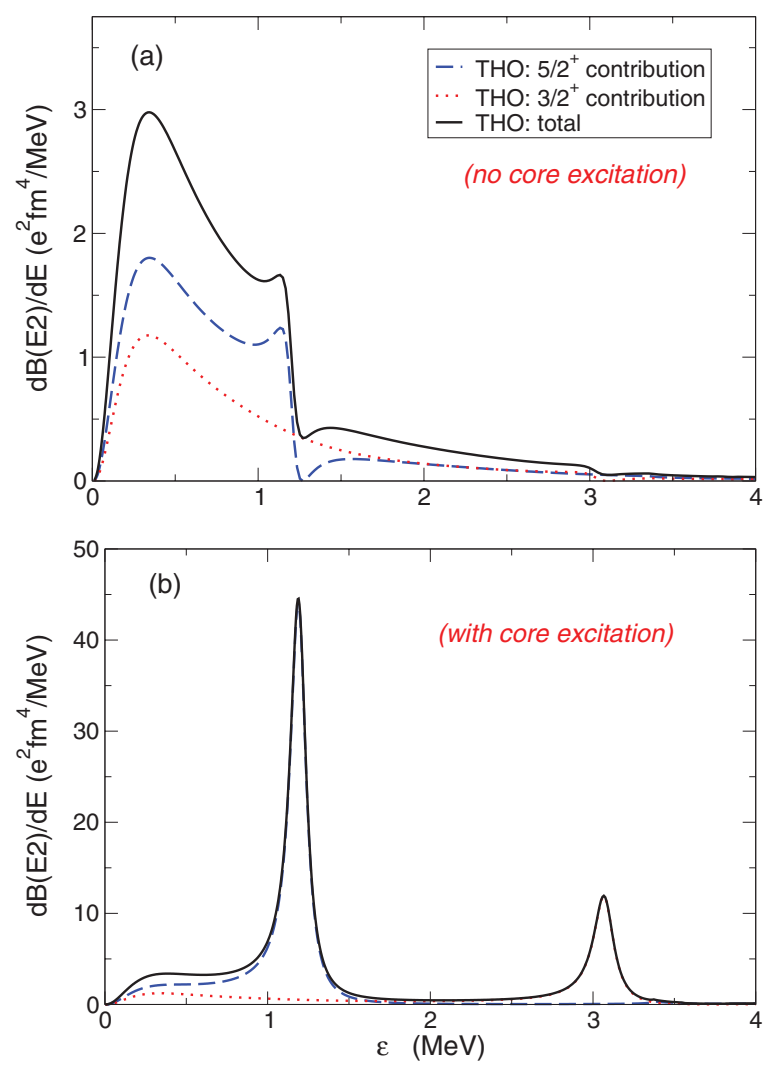

FIG. 8. (Color online) Quadrupole electric transition probability $\mathcal{B}(E 2)$ obtained with the THO basis with $N=20$ oscillator functions. The upper panel is the calculation including only the valence excitations. The bottom panel includes both valence and core contributions. 
Because only the single-particle excitation term of Eq. (16) contributes to this dipole transition, this observable can be also well reproduced within a single-particle model of ${ }^{11} \mathrm{Be}$, with the ${ }^{10} \mathrm{Be}$ core in its ground state, and including the appropriate spectroscopic factor for the $\left|{ }^{10} \mathrm{Be}\left(0^{+}\right) \otimes 2 s_{1 / 2}\right\rangle$ configuration. A departure from this behavior is the aforementioned reduction of the $\mathcal{B}(E 1)$ around $2.8 \mathrm{MeV}$, which is due to a core-dominated $3 / 2^{-}$resonance.

We have also evaluated the quadrupole electric transition probabilities, which are shown in Fig. 8. The dotted and dashed lines are the contributions from $3 / 2^{+}$and $5 / 2^{+}$, respectively, whereas the solid line is the sum of both contributions. According to Eq. (16), in addition to the single-particle excitations, in this case we have also a contribution due to $E 2$ transitions of the core which, in fact, give the main contribution to the total $\mathcal{B}(E 2)$ strength. To illustrate better the contribution coming from the valence excitation and the core, we show in the upper panel of this figure the single-particle contribution, whereas in the bottom panel we show the full calculation, including also contributions from the core. It is seen that the $\mathcal{B}(E 2)$ strength is dominated by the core excitations, as expected for a collective transition. The peaks at $\varepsilon \simeq 1.2 \mathrm{MeV}$ and and $\varepsilon \simeq 3.2 \mathrm{MeV}$ are due to the $5 / 2^{+}$and $3 / 2^{+}$resonances. Unfortunately, no experimental or theoretical $\mathcal{B}(E 2)$ for ${ }^{11} \mathrm{Be}$ has been found in the literature with which to compare.

\section{SUMMARY AND CONCLUSIONS}

We have investigated the problem of the description of the states of a particle moving in a deformed potential in terms of a pseudostate (PS) basis. In the PS method, the states of the system are approximated by the eigenstates of the Hamiltonian in a basis of square-integrable functions. The negative eigenvalues are identified with the bound states of the system, whereas the positive eigenvalues are regarded as a discrete and finite representation of the continuum spectrum. Identification of resonances is done using the socalled stabilization method [21,22].

Following our previous choice for nondeformed systems, we propose to use as PS basis the transformed harmonic oscillator (THO) basis. The basis functions are obtained by applying an analytic local-scale transformation $[5,19]$ to the conventional $\mathrm{HO}$ basis. The transformation is such that it converts the Gaussian asymptotic behavior of the $\mathrm{HO}$ function into an exponential.
The method has been applied to the ${ }^{11} \mathrm{Be}$ nucleus, treated within a particle-rotor model. The ${ }^{10} \mathrm{Be}$ core is assumed to have a permanent axial deformation with $\beta_{2}=0.67$ [18]. We have shown that the bound-state energies and wave functions are very well described using a relatively small basis, showing perfect agreement with those obtained by direct integration of the Schrödinger equation. We have shown that the resonances $5 / 2^{+}, 3 / 2^{-}$, and $3 / 2^{+}$are also well described with the method using small THO bases. It has also been checked that the wave functions of the nonresonant continuum calculated with the THO method compare well with the state computed by direct integration of the Schrödinger equation at the same energy.

We have given expressions for the $E 1$ and $E 2$ electric transition probabilities in the discrete basis, and we have proposed a method to obtain smooth distributions from these discrete values. To illustrate this method, we have calculated the $\mathcal{B}(E 1)$ and $\mathcal{B}(E 2)$ electric transition probabilities for the ${ }^{11} \mathrm{Be}$ nucleus. These distributions show a fast convergence rate with the basis size, and the converged results are in perfect agreement with the exact calculation, obtained with the exact scattering states. With the adopted Hamiltonian, the calculated $\mathcal{B}(E 1)$ distribution is consistent, but somewhat larger, than the experimental data from MSU [40].

We conclude that the THO basis provides a suitable representation to describe two-body composite systems (bound and unbound states) including the core deformation. This study provides the needed test for accomplishing a similar study for more interesting cases, such as three-body composite systems including core deformation or three-body scattering problems (two-body projectile plus a target) including dynamic core excitation. Work toward this direction is in progress.

\section{ACKNOWLEDGMENTS}

We are grateful to Ian Thompson for his help in the calculation of the multichannel scattering states. This work has been partially supported by the Spanish Ministerio de Ciencia e Innovación and FEDER funds under Projects No. FIS2011-28738-c02-01, No. FPA2009-07653, and No. FPA2009-08848, by the Spanish Consolider-Ingenio 2010 Programme CPAN (CSD2007-00042), and by Junta de Andalucía (FQM160, P07-FQM-02894). J.A.L. acknowledges a research grant by the Ministerio de Ciencia e Innovación.
[1] G. H. Rawitscher, Phys. Rev. C 9, 2210 (1974).

[2] N. Austern, Y. Iseri, M. Kamimura, M. Kawai, G. Rawitscher, and M. Yahiro, Phys. Rep. 154, 125 (1987).

[3] T. Matsumoto, T. Kamizato, K. Ogata, Y. Iseri, E. Hiyama, M. Kamimura, and M. Yahiro, Phys. Rev. C 68, 064607 (2003).

[4] F. Pérez-Bernal, I. Martel, J. M. Arias, and J. Gómez-Camacho, Few-Body Syst. Suppl. 13, 217 (2002).

[5] A. M. Moro, J. M. Arias, J. Gómez-Camacho, and F. PérezBernal, Phys. Rev. C 80, 054605 (2009).

[6] T. Matsumoto, E. Hiyama, M. Yahiro, K. Ogata, Y. Iseri, and M. Kamimura, Nucl. Phys. A 738, 471 (2004).
[7] T. Matsumoto, E. Hiyama, K. Ogata, Y. Iseri, M. Kamimura, S. Chiba, and M. Yahiro, Phys. Rev. C 70, 061601(R) (2004).

[8] T. Matsumoto, T. Egami, K. Ogata, Y. Iseri, M. Kamimura, and M. Yahiro, Phys. Rev. C 73, 051602(R) (2006).

[9] M. Rodríguez-Gallardo, J. M. Arias, J. Gómez-Camacho, R. C. Johnson, A. M. Moro, I. J. Thompson, and J. A. Tostevin, Phys. Rev. C 77, 064609 (2008).

[10] K. Hagino and N. Van Giai, Nucl. Phys. A 735, 55 (2004).

[11] N. C. Summers, F. M. Nunes, and I. J. Thompson, Phys. Rev. C 74, 014606 (2006). 
[12] R. Crespo, A. Deltuva, and A. M. Moro, Phys. Rev. C 83, 044622 (2011).

[13] A. M. Moro and R. Crespo, Phys. Rev. C 85, 054613 (2012).

[14] D. Vautherin, Phys. Rev. C 7, 296 (1973).

[15] Y. K. Gambhir, P. Ring, and A. Thimet, Ann. Phys. (NY) 198 132 (1990).

[16] S. G. Zhou, J. Meng, and P. Ring, Phys. Rev. C 68, 034323 (2003).

[17] J. M. Bang and J. S. Vaagen, Z. Phys. A 297, 223 (1980).

[18] F. Nunes, J. Christley, I. Thompson, R. Johnson, and V. Efros, Nucl. Phys. A 609, 43 (1996).

[19] S. Karataglidis, K. Amos, and B. G. Giraud, Phys. Rev. C 71, 064601 (2005).

[20] J. A. Lay, A. M. Moro, J. M. Arias, and J. Gómez-Camacho, Phys. Rev. C 82, 024605 (2010).

[21] A. U. Hazi and H. S. Taylor, Phys. Rev. A 1, 1109 (1970).

[22] H. S. Taylor and A. U. Hazi, Phys. Rev. A 14, 2071 (1976).

[23] M. V. Stoitsov and I. Z. Petkov, Ann. Phys. (NY) 184, 121 (1988).

[24] I. Z. Petkov and M. V. Stoitsov, Nuclear Density Functional Theory, Oxford Studies in Physics (Clarendon, Oxford, 1991).

[25] F. Pérez-Bernal, I. Martel, J. M. Arias, and J. Gómez-Camacho, Phys. Rev. A 63, 052111 (2001).
[26] J. A. Tostevin, F. M. Nunes, and I. J. Thompson, Phys. Rev. C 63, 024617 (2001).

[27] A. Bohr and B. Mottelson, Nuclear Structure (W. A. Benjamin, New York, 1969).

[28] D. M. Brink and G. R. Satchler, Angular Momentum (Clarendon, Oxford, 1968).

[29] J. A. Lay, D. V. Fedorov, A. S. Jensen, E. Garrido, and C. Romero-Redondo, Eur. Phys. J. A 44, 261 (2010).

[30] S. Typel and G. Baur, Nucl. Phys. A 759, 247 (2005).

[31] A. M. Moro, F. Pérez-Bernal, J. M. Arias, and J. GómezCamacho, Phys. Rev. C 73, 044612 (2006).

[32] P. Capel, G. Goldstein, and D. Baye, Phys. Rev. C 70, 064605 (2004).

[33] D. J. Howell, J. A. Tostevin, and J. S. Al-Khalili, J. Phys. G 31, S1881 (2005).

[34] S. Fortier et al., Phys. Lett. B 461, 22 (1999).

[35] J. S. Winfield et al., Nucl. Phys. A 683, 48 (2001).

[36] F. Cappuzzello, A. Cunsolo, S. Fortier, A. Foti, M. Khaled, H. Laurent, H. Lenske, J. M. Maison, A. L. Melita, C. Nociforo et al., Phys. Lett. B 516, 21 (2001).

[37] B. A. Lippmann and T. F. O’Malley, Phys. Rev. A 2, 2115 (1970).

[38] F. Nunes, Ph.D. thesis, University of Surrey, 1995 (unpublished).

[39] T. Nakamura et al., Nucl. Phys. A 588, c81 (1995).

[40] R. Palit et al., Phys. Rev. C 68, 034318 (2003). 